\title{
Editorial
}

\section{Mathematical Modeling of Marine Structures}

\author{
Carl M. Larsen, ${ }^{1}$ Armin Troesch, ${ }^{2}$ Celso P. Pesce, ${ }^{3}$ \\ and Ioannis K. Chatjigeorgiou ${ }^{4}$ \\ ${ }^{1}$ Department of Marine Technology, Norwegian University of Science and Technology, 7491 Trondheim, \\ Norway \\ ${ }^{2}$ Department of Naval Architecture and Marine Engineering, University Michigan, Ann Arbor, MI 48 109, \\ USA \\ ${ }^{3}$ Department of Mechanical Engineering and Graduate Program in Ocean Engineering, University of São \\ Paulo, 05508-900, São Paulo, SP, Brazil \\ ${ }^{4}$ Department of Naval Architecture and Marine Engineering, National Technical University of Athens, \\ 10682 Athens, Greece
}

Correspondence should be addressed to Carl M. Larsen, carl.m.larsen@ntnu.no

Received 17 December 2012; Accepted 17 December 2012

Copyright (C) 2012 Carl M. Larsen et al. This is an open access article distributed under the Creative Commons Attribution License, which permits unrestricted use, distribution, and reproduction in any medium, provided the original work is properly cited.

Naval architecture and ocean engineering deal with design and operation of a large variety of structures for use at sea. Striving for cost effective and safe designs has resulted in development of sophisticated methods for predicting the behaviour of these structures. The core disciplines have been oceanography, hydrodynamics, dynamics, structural mechanics, and material science, but recently we have also seen an increasing use of cybernetics and information technology. Applied mathematics is the common tool we have when applying the basic disciplines on our practical problems.

This special issue attracted a large interest, mainly from academic institutions dealing with marine, civil and mechanical engineering, but also applied mathematics. More than 50 papers were submitted, and 22 of these were accepted for publication.

The largest group of accepted papers deals with marine hydrodynamics. Various aspects of ocean wave theories are discussed in five papers. Key words to describe the content are shallow water, tsunamis, modulation instabilities of wave systems, numerical wave tank, and use of particle finite element models. Two papers deal with sloshing in tanks and discussions of rigid body motions of floating bodies are found in two papers.

Hydroelasticity means that dynamic simulations require an integrated calculation of elastic deformations and hydrodynamic forces. Three papers fall into this category.

Dynamic response of marine structures is another important discipline in marine technology, and six papers are found in this category. Collision between ships and other types of elastic structures is analysed in two papers. Flexible risers and umbilicals represent 
a special type of marine structures. A global analysis must normally be carried out by use of nonlinear time domain methods, but the global model will normally not include a detailed model of all elements in a cross section. The cross section will often consist of spiral elements, small diameter pipes, and concentric plastic pipes. Local stress analysis must therefore account for sliding between individual substructures and hence also friction forces and is therefore often carried out by postprocessing of results from the global analysis. Four papers present methods for this type of calculations.

Other aspects of slender marine structures are presented in two papers. One deals with optimization of a slender pipe as a key element in a deep sea mining system, while a method that accounts for the effects of geometrical and material data on the collapse pressure of inhomogeneous rings under external hydrostatic pressure is the topic of the second paper.

Finally, three papers deal with specific topics that do not belong to the above categories, namely, oil spill simulation, a parabolic partial differential equation describing the thermodynamic field of sea ice, and finally simulation of the installation of torpedo anchors.

Carl M. Larsen

Armin Troesch

Celso P. Pesce

Ioannis K. Chatjigeorgiou 


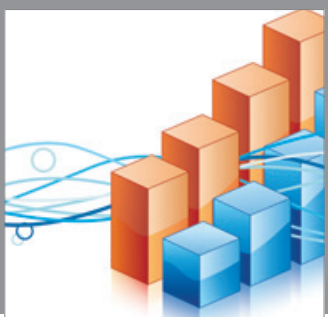

Advances in

Operations Research



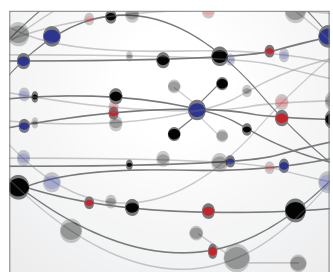

\section{The Scientific} World Journal
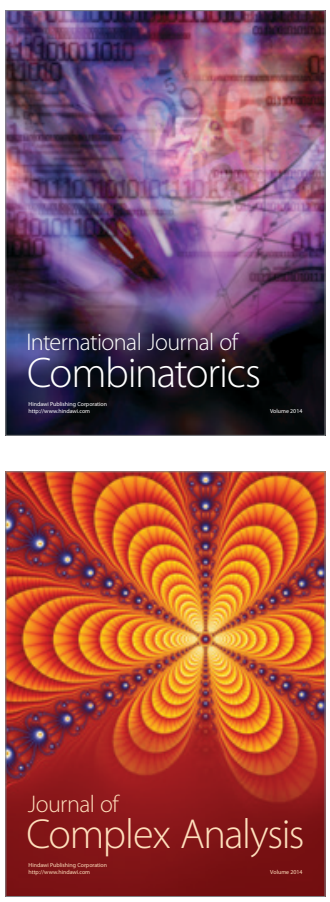

International Journal of

Mathematics and

Mathematical

Sciences


Journal of

Applied Mathematics
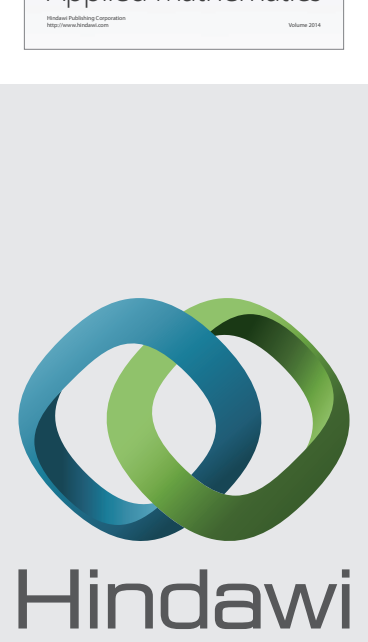

Submit your manuscripts at http://www.hindawi.com
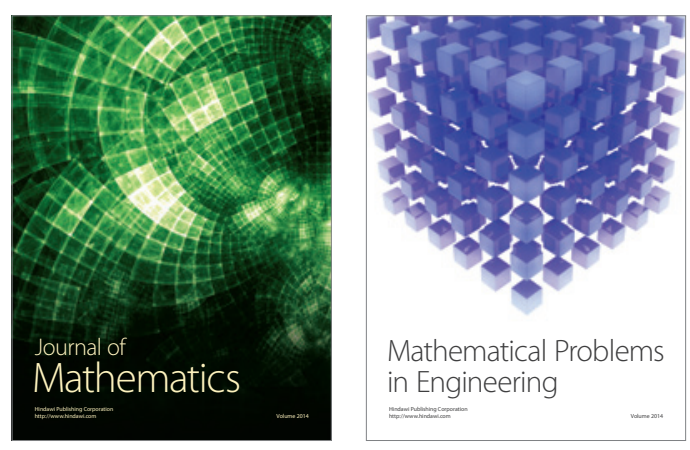

Mathematical Problems in Engineering
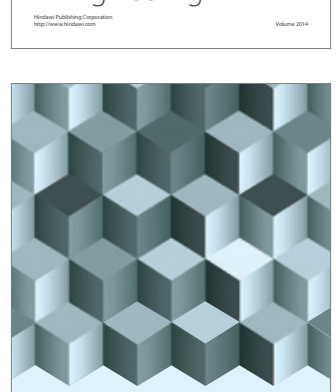

Journal of

Function Spaces
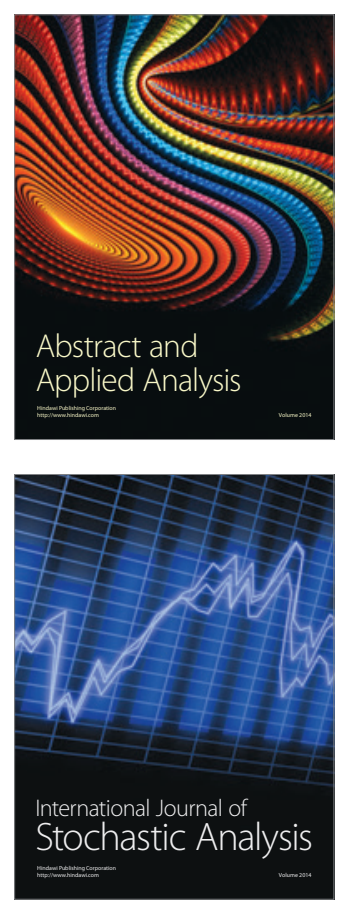

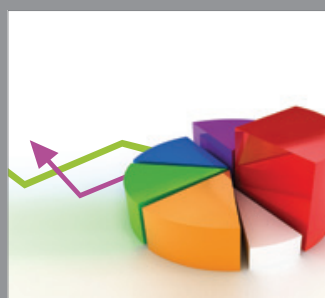

ournal of

Probability and Statistics

Promensencen
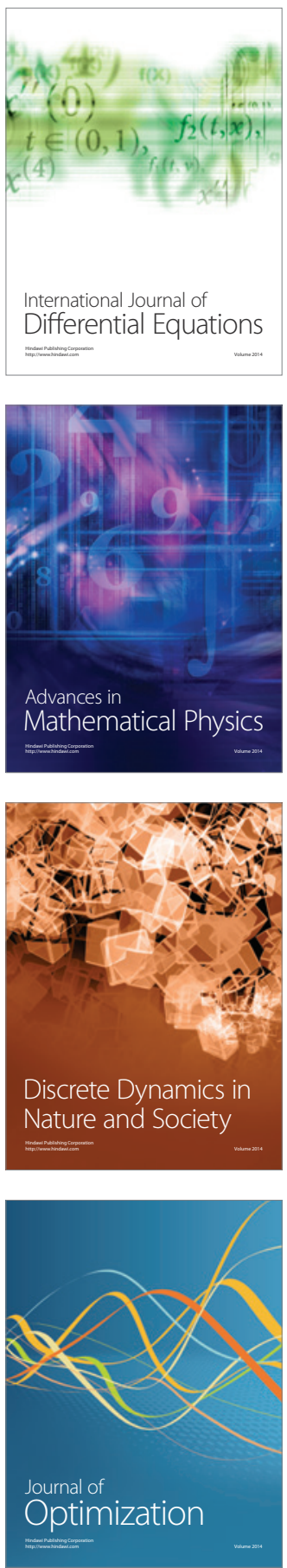